\title{
General fifth M-Zagreb indices and fifth $M$ - Zagreb polynomials of carbon graphite
}

\begin{abstract}
Abdul Qudair Baiga ${ }^{\bigcirc} \mid$ Muhammad Naeem ${ }^{\mathrm{b}, *}{ }^{\circ} \mid$ Wei Gaoc $\mid$ |Jia-Bao Liud
aDepartment of Mathematics and Statistics, Institute of Southern Punjab, Multan, Pakistan

${ }^{b}$ Department of Mathematics, The University of Lahore, Pakpattan Campus, Pakistan

cSchool of Information Science and Technology, Yunnan Normal University, Kunming, China

${ }^{d}$ School of Mathematics and Physics, Anhui Jianzhu University, Hefei 230601, P.R. China

Graph theory plays a vital role in modeling and designing any chemical structure or chemical network. Chemical graph theory helps to understand the molecular structural properties of a molecular graph. The molecular graph is a graph consisting of atoms called vertices and the chemical bond between atoms called edges. In this paper, we have computed the various types of degree-based fifth M-Zagreb indices, general fifth M-Zagreb indices, fifth hyper-M-Zagreb indices, general fifth $\mathrm{M}_{1}$-Zagreb polynomial, general fifth $\mathrm{M}_{2}$-Zagreb polynomial, fifth $\mathrm{M}_{1}$-Zagreb polynomial and fifth $\mathrm{M}_{2}$-Zagreb polynomial, fifth hyper- $\mathrm{M}_{1-}$ Zagreb polynomial, fifth hyper-M2-Zagreb polynomial and fifth $\mathrm{M}_{3}$-Zagreb index and their polynomials of a molecular graph namely carbon graphite denoted by $C G(m, n)$ for t-levels.
\end{abstract}

\section{KEYWORDS}

General fifth M-Zagreb indices; general fifth M-Zagreb polynomials; carbon graphite.

\section{*Corresponding Author: \\ Muhammad Naeem \\ Email: naeempkn@gmail.com Tel.: +92 3234361991}

\section{Introduction}

Let $\mathrm{G}=(V, \mathrm{E})$ be a graph where $\mathrm{V}$ be the vertex set and $\mathrm{E}$ be the edge set of $\mathrm{G}$. A chemical graph can be represented by a polynomial, a numerical value or matrix form. In a chemical graph, vertex of graph represents the atoms and the edges represent the bonds between the atoms of the compound under study. Over the years, the mathematicians and the people working in mathematical chemistry have computed and used certain types of numerical numbers related to chemical graph to discuss and study the properties of chemical graph. The topological indices mainly eccentric-based, degree-based and distance-based indices etc. lie in the core of this study. In this article, we deal with some old and newly introduced degree-based topological indices.

Let $\mathrm{S}_{\mathrm{G}}(\mathrm{u})$ denote the sum of the degrees of all vertices adjacent to a vertex $u$. Graovac et al. defined fifth M-Zagreb indices as polynomials for a molecular graph [4] and these are characterized as

$$
\begin{gathered}
M_{1} G_{5}(G)=\sum_{u v \in E(G)}\left(S_{G}(u)+S_{G}(v)\right), \\
M_{2} G_{5}(G)=\sum_{u v \in E(G)}\left(S_{G}(u) S_{G}(v)\right)
\end{gathered}
$$

Motivated by above indices, V.R. Kulli introduced the general fifth $M$-Zagreb indices, fifth M-Zagreb in [8] and fifth hyper-MZagreb indices, which are characterized as

$$
\begin{gathered}
M_{1}^{a} G_{5}(G)=\sum_{u v \in E(G)}\left(S_{G}(u)+S_{G}(v)\right)^{a}, \\
M_{2}^{a} G_{5}(G)=\sum_{u v \in E(G)}\left(S_{G}(u) S_{G}(v)\right)^{a} \\
H M_{1} G_{5}(G)=\sum_{u v \in E(G)}\left(S_{G}(u)+S_{G}(v)\right)^{2}, \\
H M_{2} G_{5}(G)=\sum_{u v \in E(G)}\left(S_{G}(u) S_{G}(v)\right)^{2}
\end{gathered}
$$

They also define a new version of Zagreb index which they call as third Zagreb index or fifth $\mathrm{M}_{3}$-Zagreb index [5],

$M_{3} G_{5}(G)=\sum_{u v \in E(G)}\left|S_{G}(u)-S_{G}(v)\right|$

Corresponding the above indices, they defined the general fifth $\mathrm{M}_{1}$-Zagreb 
polynomial and the general fifth $\mathrm{M}_{2}$-Zagreb polynomial of a molecular graph $\mathrm{G}$ as

$$
\begin{aligned}
M_{1}^{a} G_{5}(G, x) & =\sum_{u v \in E(G)} x^{\left(s_{G}(u)+S_{G}(v)\right)^{a},} \\
M_{2}^{a} G_{5}(G, x) & =\sum_{u v \in E(G)} x^{\left(S_{G}(u) S_{G}(v)\right)^{a}}
\end{aligned}
$$

The fifth $\mathrm{M}_{1}$-Zagreb polynomial and fifth $\mathrm{M}_{2}$ Zagreb polynomial of a molecular graph $\mathrm{G}$ are defined as

$$
\begin{gathered}
M_{1} G_{5}(G, x)=\sum_{u v \in E(G)} x^{\left(s_{G}(u)+S_{G}(v)\right),} \\
M_{2} G_{5}(G, x)=\sum_{u v \in E(G)} x^{\left(S_{G}(u) S_{G}(v)\right)}
\end{gathered}
$$

The fifth hyper- $\mathrm{M}_{1}$-Zagreb polynomial and fifth hyper- $\mathrm{M}_{2}$-Zagreb polynomial of a molecular graph $\mathrm{G}$ are defined as;

$$
\begin{gathered}
M_{1} G_{5}(G, x)=\sum_{u v \in E(G)} x^{\left(S_{G}(u)+S_{G}(v)\right)^{2}}, \\
M_{2} G_{5}(G, x)=\sum_{u v \in E(G)} x^{\left(S_{G}(u) S_{G}(v)\right)^{2}}
\end{gathered}
$$

For further study and literature related to the topological indices, see references [2], [3], [5], [7], [8], [10], [11], [13], and [14].

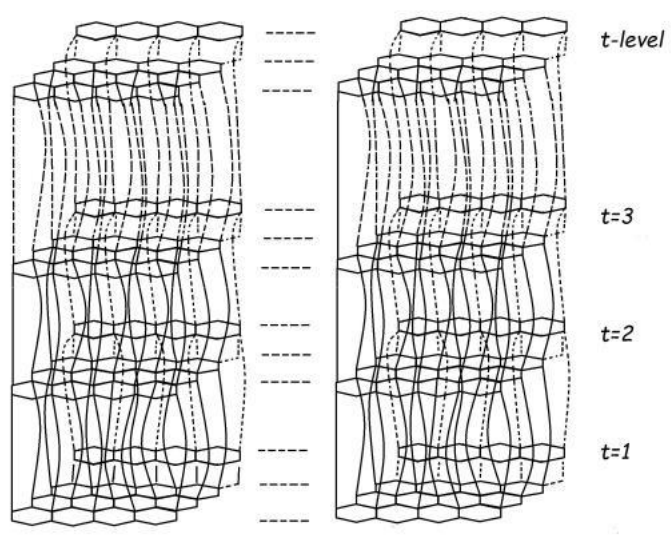

FIGURE 1Carbon graphite $C G(m, n)$ t-levels

\section{Main results}

In this paper, we have computed the general fifth $\mathrm{M}$-Zagreb indices and fifth $\mathrm{M}_{3}$-Zagreb index and their polynomials of a molecular graph namely carbon graphite denoted by $C G(m, n)$ for t-levels. Graphite is an allotrope of carbon. The chemical graph of carbon graphite $C G(m, n)$ consists of a hexagon shapes. The structure of carbon graphite consists of hexagons in the form of layers one after the other and there is a weak bond between these layers. The molecular graph of carbon graphite $C G(m, n)$ for t-levels is depicted in Figure 1 . The cardinality of vertices and edges in $C G(m, n)$ are $2 \mathrm{t}(\mathrm{mn}+\mathrm{m}+\mathrm{n})$ and $4 \mathrm{mnt}+3 \mathrm{mt}+2 \mathrm{nt}-\mathrm{mn}-\mathrm{m}-\mathrm{t}$ respectively. A single layer in carbon graphite depicts the graphene layer. Carbon graphite consists of multiple layers of graphene. The $t$ is the level that depicts graphene layers in carbon graphite, $\mathrm{m}$ is the number of rows and $\mathrm{n}$ is the number of columns in each layer with $\mathrm{m}$ taken as $\mathrm{n}$ copies of hexagons in a row and $\mathrm{n}$ is taken as $\mathrm{m}$ copies of hexagons in the column, where in each level or layer, the hexagons from left to right are defined as the hexagons in row and the hexagons from down to up direction are defined as the hexagons in the column. In Figure 2, the levels of carbon graphite is $t=3, m=3$ and $n=4$, where $m=3$ is taken as $n=4$ copies of hexagons in a row in each level $t=3$ and $n=4$ is taken as $m=3$ copies of hexagons in the column in each level.

In $C G(m, n)$, the 2 degree vertices are $2(n t+m+1)$, the 3 degree vertices are $2 m n+2 m t-2 m+2 t-4$ and 4 degree vertices are $2(m n-1)(t-1)$. Some literature about the degree-based topological indices of carbon graphite $C G(m, n)$ can be seen in $[1,5]$.

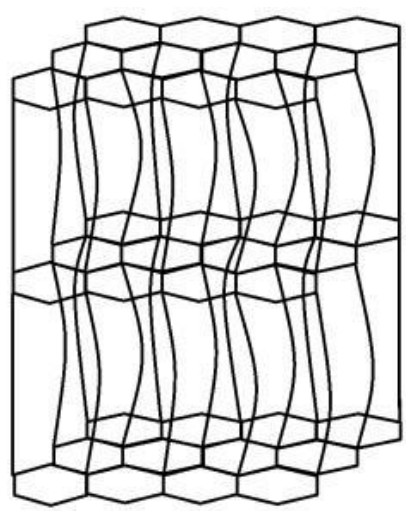

FIGURE 2 Carbon graphite graph for $(3,4)$ and $\mathrm{t}=3$ 
Table 1 shows the edge partition based on the compute fifth M-Zagreb indices using Table 1. degree sum of end vertices of each edge. We

TABLE 1Edge partition of $C G(m, n)$ basedon degree sum of end vertices of each edge

\begin{tabular}{cccc}
\hline$\left(\mathbf{S}_{\mathbf{u}}, \mathbf{S}_{\mathbf{v}}\right)$ & Frequency & $\left(\mathbf{S}_{\mathbf{u}}, \mathbf{S}_{\mathbf{v}}\right)$ & Frequency \\
\hline$(5,5)$ & 2 & $(8,12)$ & $4(\mathrm{nt}-2 \mathrm{n}-2 \mathrm{t}+4)$ \\
$(5,6)$ & 2 & $(8,13)$ & $2(\mathrm{n}-1)$ \\
$(5,8)$ & 6 & $(9,10)$ & 2 \\
$(6,8)$ & $4(\mathrm{n}-2)$ & $(9,13)$ & 2 \\
$(6,10)$ & 2 & $(10,10)$ & $4 \mathrm{~m}-6$ \\
$(7,7)$ & 4 & $(10,12)$ & $4 \mathrm{~m}-6$ \\
$(7,8)$ & $2(\mathrm{t}-2)$ & $(10,13)$ & $4 \mathrm{~m}+6 \mathrm{t}-20$ \\
$(7,9)$ & 2 & $(11,12)$ & $2(\mathrm{n}-1)$ \\
$(7,10)$ & $2 \mathrm{t}$ & $(11,16)$ & $2(\mathrm{n}-1)$ \\
$(7,11)$ & 4 & $(12,13)$ & $4 \mathrm{nt}-10 \mathrm{n}-4 \mathrm{t}+10$ \\
$(7,12)$ & $4(\mathrm{t}-2)$ & $(12,16)$ & $2 \mathrm{mn}+2 \mathrm{mt}-6 \mathrm{~m}-4 \mathrm{n}-2 \mathrm{t}+8$ \\
$(8,8)$ & 2 & $(13,16)$ & \\
$(8,10)$ & $4(\mathrm{~m}+\mathrm{t}-4)$ & $(16,16)$ & $4 \mathrm{mnt}-5 \mathrm{mt}-6 \mathrm{nt}-9 \mathrm{mn}+11 \mathrm{~m}+14 \mathrm{n}+7 \mathrm{t}-16$ \\
$(8,11)$ & $4(\mathrm{n}-2)$ & & \\
\hline
\end{tabular}

Theorem 2.1. Consider the graph of carbon graphite $C G(m, n)$ with $\mathrm{m} ; \mathrm{n}>2$ and $t \geq 3$, then general fifth $M_{1}$-Zagreb index is equal to $\mathrm{M}_{1} \mathrm{a}_{5}(\mathrm{G})=(4 \mathrm{mnt}-5 \mathrm{mt}-6 \mathrm{nt}-9 \mathrm{mn}+$ $11 m n+11 m+14 n+7 t-16) 32^{a}+(6 m n+$ $6 m t-22 m-6 n-12 t+36) 25^{a}+(2 m n+2 m t-$ $6 m-4 n-2 t+8) 29 a+(4 m-8 n-8 t+4 n t+10)$ $20^{a}+(4 m+2 n+6 t-22) 23^{a}+(4 n t-10 n-4 t+$ 10) $28^{a}+(4 m+4 t-12) 18^{a}+(4 n+4 t-14)$ $19^{a}+(4 n-4) 14^{a}+(2 t-4) 15^{a}+(2 n-$ 2) $21^{a}+(4 m-4) 22^{a}+(2 n-$

2) $27^{\mathrm{a}}+2 \mathrm{t} \times 17^{\mathrm{a}}+2 \times 11^{\mathrm{a}}+6 \times 13 \mathrm{a}+6 \times 16 \mathrm{a}+2 \times 10 \mathrm{a}$.

Proof. Let $\mathrm{G} \approx C G(m, n)$ be a graph of carbon graphite. The above result can be proved by using Table1 and Equation 2 in the following computation. The general fifth $\mathrm{M}_{1} \mathrm{a}(\mathrm{G})$-Zagreb index is computed as below:

$\operatorname{M1Ag5}(\mathrm{G})=\sum_{u v \in E(G)}\left(S_{\left.G^{(} u\right)}+\left(S_{G^{(v)}}\right)^{\mathrm{a}}=(2)(5+\right.$ $5)^{\mathrm{a}}+(9+10)^{\mathrm{a}}+(4)(7+7)^{\mathrm{a}}+2(\mathrm{t}-2)(7+8)^{\mathrm{a}}+$ $(2)(7+9)^{a}+(2 t)(7+10)^{a}+(4)(7+11)^{a}+4(t-$ $2)(7+12)^{\mathrm{a}}+(2)(8+8)^{\mathrm{a}}+4(\mathrm{~m}+\mathrm{t}-4)(8+$ $10)^{a}+4(n-2)(8+11)^{a}+4(n t-2 n-2 t+4)(8$ $+12)^{\mathrm{a}}+2(\mathrm{n}-1)(8+13)^{\mathrm{a}}+$ $(2)+(9+10)^{\mathrm{a}}+(2)(9+13)^{\mathrm{a}}+(4 \mathrm{~m}-$

6) $(10+10)^{a}+(4 m-6)(10+12)^{a}+(4 m+6 t-$

$20)(10+13)^{a}+2(n-1)(11+12)^{a}+2(n-1)(11+$ $16)^{a}+(6 m n+6 m t-22 m-6 n-12 t+36)(12+$ $13)^{a}+(4 n t-10 n-4 t+10)(12+16)^{a}+(4 m n t$ $-5 m t-6 n t-9 m n+11 m+14 n+7 t-16)(16+$ $16)^{a}+(2 m n+2 m t-6 m-4 n-2 t+8)(13+16)^{a}$ After simplification, we got:

$$
\begin{aligned}
& M_{1}^{a} G_{5}(G)=(4 m n t-5 m t-6 n t-9 m n+11 m n+11 m \\
& +14 n+7 t-16) 32^{a}+(6 m n+6 m t-22 m-6 n-12 t \\
& +36) 25^{a}+(2 m n+2 m t-6 m-4 n-2 t+8) 29^{a}+(4 m \\
& -8 n-8 t+4 n t+10) 20^{a}+(4 m+2 n+6 t-22) 23^{a}+(4 n t \\
& -10 n-4 t+10) 28^{a}+(4 m+4 t-12) 18^{a}+(4 n+4 t \\
& -14) 19^{a}+(4 n-4) 14^{a}+(2 t-4) 15^{a}+(2 n-2) 21^{a} \\
& +(4 m-4) 22^{a}+(2 n-2) 27^{a}+2 t \times 17^{a}+2 \times 11^{a}+6 \times 13^{a} \\
& +6 \times 16^{a}+2 \times 10^{a}
\end{aligned}
$$

Corollary 2.1. Consider the graph of carbon graphite $C G(m, n)$ with $\mathrm{m} ; \mathrm{n}>2$ and $t \geq 3$, then fifth $M_{1}$-Zagreb index is obtained below replacing a $=1$ in Theorem 2.1 $M_{1} G_{5}(G)=28+16 n-56 t-80 m n+128 m n t+48 m t-$ $40 \mathrm{~m}$.

Corollary 2.2. Consider the graph of carbon graphite $C G(m, n)$ with $m ; n>2$ andt $\geq 3$, then fifth hyper-M-Zagreb index is obtained below replacing $\mathrm{a}=2$ in Theorem 2.1 
$H M_{1} G_{5}(G)=1136+1808 n-1408 t-$

$3784 m n+4096 m n t+312 m t-1408 n t-584 m$.

Theorem 2.2. Consider the graph of carbon graphite $C G(m, n)$ with $m ; n>2$ and $t \geq 3$, then general fifth $\mathrm{M}_{2}$-Zagreb index is equal to

$$
\begin{aligned}
& M_{2}^{a} G_{5}(G)=2 \times 117^{a}+2 \times 90^{a}+2 \times 64^{a} \\
& +4 \times 77^{a}+2 \times 63^{a}+4 \times 49^{a}+2 \times 60^{a}+6 \times 40^{a}+2 \times 30^{a} \\
& +2 \times 25^{a}+(2 n-2) 104^{a}+(4 t-8) 84^{a}+(4 m+4 t-16) 80^{a} \\
& +(4 n-8) 88^{a}+(4 n-8) 48^{a}+(2 t-4) 56^{a}+2 t 70^{a}+(2 m n \\
& +2 m t-6 m-4 n-2 t+8) 208^{a}+(4 m n t-5 m t-6 n t-9 m n \\
& +11 m+14 n+7 t-16) 256^{a}+(6 m n+6 m t-22 m-6 n-12 t \\
& +36) 156^{a}+(4 n t-10 n-4 t+10) 192^{a}+(2 n-2) 176^{a}+(4 m \\
& -6) 120^{a}+(4 m+6 t-20) 130^{a}+(2 n-2) 132^{a}+(4 m-6) 100^{a} \\
& +(4 n t-8 n-8 t+16) 96^{a} .
\end{aligned}
$$

Proof. Let $\mathrm{G} \approx C G(m, n)$ be a graph of carbon graphite. The above result can be proved by using Table1 and Equation 2 in the following computation. The general fifth $\mathrm{M}_{2} \mathrm{a}(\mathrm{G})$-Zagreb index is computed as below:

$$
\begin{aligned}
& M_{2}^{a} G_{5}(G)=\sum_{u v \in E(G)}\left(S_{G}(u) S_{G}(v)\right)^{a}=(2)(5 \times 5)^{a} \\
& +(2)(5 \times 6)^{a}+(6)(5 \times 8)^{a}+4(n-2)(6 \times 8)^{a}+(2) \\
& (6 \times 10)^{a}+(4)(7 \times 7)^{a}+2(t-2)(7 \times 8)^{a}+(2)(7 \times 9)^{a} \\
& +(2 t)(7 \times 10)^{a}+(4)(7 \times 1)^{a}+4(t-2)(7 \times 12)^{a}+(2) \\
& (8 \times 8)^{a}+4(m+t-4)(8 \times 10)^{a}+4(n-2)(8 \times 11)^{a} \\
& +4(n t-2 n-2 t+4)(8 \times 12)^{a}+2(n-1)(8 \times 13)^{a}+(2) \\
& (9 \times 10)^{a}+(2)(9 \times 13)^{a}+(4 m-6)(10 \times 10)^{a}+(4 m-6) \\
& (10 \times 12)^{a}+(4 m+6 t-20)(10 \times 13)^{a}+2(n-1)(11 \times 12)^{a} \\
& +2(n-1)(11 \times 16)^{a}+(6 m n+6 m t-22 m-6 n-12 t+36)(12 \\
& \times 13)^{a}+(4 n t-10 n-4 t+10)(12 \times 16)^{a}+(4 m n t-5 m t \\
& -6 n t-9 m n+11 m+14 n+7 t-16)(16 \times 16)^{a}+(2 m n+2 m t \\
& -6 m-4 n-2 t+8)(13 \times 16)^{a}
\end{aligned}
$$

An easy calculation gives us the following result:

$$
\begin{aligned}
& M_{2}^{a} G_{5}(G)=2 \times 117^{a}+2 \times 90^{a}+2 \times 64^{a} \\
& +4 \times 77^{a}+2 \times 63^{a}+4 \times 49^{a}+2 \times 60^{a}+6 \times 40^{a}+2 \times 30^{a} \\
& +2 \times 25^{a}+(2 n-2) 104^{a}+(4 t-8) 84^{a}+(4 m+4 t-16) 80^{a} \\
& +(4 n-8) 88^{a}+(4 n-8) 48^{a}+(2 t-4) 56^{a}+2 t 70^{a}+(2 m n \\
& +2 m t-6 m-4 n-2 t+8) 208^{a}+(4 m n t-5 m t-6 n t-9 m n \\
& +11 m+14 n+7 t-16) 256^{a}+(6 m n+6 m t-22 m-6 n-12 t \\
& +36) 156^{a}+(4 n t-10 n-4 t+10) 192^{a}+(2 n-2) 176^{a}+(4 m \\
& -6) 120^{a}+(4 m+6 t-20) 130^{a}+(2 n-2) 132^{a}+(4 m-6) 100^{a} \\
& +(4 n t-8 n-8 t+16) 96^{a} .
\end{aligned}
$$

Corollary 2.3. Consider the graph of carbon graphite $C G(m, n)$ with $\mathrm{m} ; \mathrm{n}>2$ andt $\geq 3$, then fifth $\mathrm{M}_{2}$-Zagreb index is obtained below replacing a $=1$ in Theorem2.2:

$M_{2} G_{5}(G)=274-952 m n+1024 m n t+72 m t-384 n t-$

\section{$144 m-344 t+496 n$.}

Corollary 2.4. Consider the graph of carbon graphite $C G(m, n)$ with $m ; n>2$ andt $\geq 3$, then fifth hyper- $\mathrm{M}_{2}$-Zagreb index is obtained below replacing $\mathrm{a}=2$ in Theorem 2.2 $H_{2} G_{5}(G)=-52002-357280 m n+262144 m n t-$ $95136 m t-$

$208896 n t+116720 m+30304 t+314688 n$.

Theorem 2.3. Consider the graph of carbon graphite $C G(m, n)$ with $m ; n>2$ andt $\geq 3$, then fifth $\mathrm{M}_{3}$-Zagreb index is equal to: $M_{3} G_{5}(G)=12-48 n+12 m n-12 t+12 m t+32 n t-12 m$. Proof. Let $\mathrm{G} \approx C G(m, n)$ be a graph of carbon graphite. The above result can be proved using Table 1 and Equation 4 in the following computation. The fifth $\mathrm{M}_{3} \mathrm{G}_{5}(\mathrm{G})$-Zagreb index is computed as below: 


$$
\begin{aligned}
& \left.M_{3} G_{5}(G)=\sum_{u v \in E(G)} \mid S_{G}(u)-S_{G}(v)\right)|=(2)| 5-5 \mid \\
& +(2)|5-6|+(6)|5-8|+4(n-2)|6-8|+(2) \\
& |6-10|+(4)|7-7|+2(t-2)|7-8|+(2)|7-9| \\
& +(2 t)|7-1|+(4)|7-11|+4(t-2)|7-12|+(2) \\
& |8-8|+4(m+t-4)|8-10|+4(n-2)|8-11| \\
& +4(n t-2 n-2 t+4)|8-12|+2(n-1)|8-13|+(2)|9-10| \\
& +(2)|9-13|+(4 m-6)|10-10|+(4 m-6) \\
& |10-12|+(4 m+6 t-20)|10-13|+2(n-1)|11-12| \\
& +2(n-1)|11-16|+(6 m n+6 m t-22 m-6 n-12 t+36) \mid 12 \\
& -13|+(4 n t-10 n-4 t+10)| 12-16 \mid+(4 m n t-5 m t \\
& -6 n t-9 m n+11 m+14 n+7 t-16)|16-16|+(2 m n+2 m t \\
& -6 m-4 n-2 t+8)|13-16|
\end{aligned}
$$

After computation, we obtain:

$$
M_{3} G_{5}(G)=12-48 n+12 m n-12 t+12 m t+32 n t-12 m \text {. }
$$

Theorem 2.4. Consider the graph of carbon graphite $C G(m, n)$ with $m ; n>2$ and $t \geq 3$, then general fifth $M_{1}$-Zagreb polynomial is equal to:

$$
\begin{aligned}
& M_{1}^{a} G_{5}(G, x)=(2 m n+2 m t-6 m-4 n-2 t+8) x^{29^{a}}+(4 m n t \\
& -5 m t-6 n t-9 m n+11 m+14 n+7 t-16) x^{32^{a}}+(4 n t-10 n-4 t \\
& +10) x^{28^{a}}+(6 m n+6 m t-22 m-6 n-12 t+36) x^{25^{a}}+(2 n-2) x^{23^{a}} \\
& +(4 m-6) x^{22^{a}}(4 m+6 t-20) x^{23^{a}}+(4 m-6) x^{20^{a}}+(4 n-8) x^{19^{a}} \\
& +(4 n t-8 n-8 t+16) x^{20^{a}}+(4 n-8) x^{14^{a}}+(2 t-4) x^{15^{a}}+2 t x^{17^{a}} \\
& +6 x^{16^{a}}+6 x^{13^{a}}+2 x^{11^{a}}+2 x^{10^{a}}+2 x^{22^{a}}+2 x^{19^{a}}+4 x^{18^{a}}+4 x^{14^{a}} .
\end{aligned}
$$

Proof. Let $\mathrm{G} \approx C G(m, n)$ be a graph of carbon graphite. The above result can be proved using Table 1 and Equation 5 in the following computation. The general fifth $\mathrm{M}^{\mathrm{a}}(\mathrm{G}, \mathrm{x})$ Zagreb polynomial is computed as below:

$$
\begin{aligned}
& M_{1}^{a} G_{5}(G, x)=\sum_{u v \in E(G)} x^{\left(S_{G}(u)+S_{G}(v)\right)^{a}}=(2) x^{(5+5)^{a}} \\
& +(2) x^{(5+6)^{a}}+(6) x^{(5+8)^{a}}+4(n-2) x^{(6+8)^{a}}+(2) \\
& x^{(6+10)^{a}}+(4) x^{(7+7)^{a}}+2(t-2) x^{(7+8)^{a}}+(2) x^{(7+9)^{a}} \\
& +(2 t) x^{(7+10)^{a}}+(4) x^{(7+11)^{a}}+4(t-2) x^{(7+12)^{a}}+(2) \\
& x^{(8+8)^{a}}+4(m+t-4) x^{(8+10)^{a}}+4(n-2) x^{(8+11)^{a}} \\
& +4(n t-2 n-2 t+4) x^{(8+12)^{a}}+2(n-1) x^{(8+13)^{a}}+(2) \\
& x^{(9+10)^{a}}+(2) x^{(9+13)^{a}}+(4 m-6) x^{(10+10)^{a}}+(4 m-6) \\
& x^{(10+12)^{a}}+(4 m+6 t-20) x^{(10+13)^{a}}+2(n-1) x^{(11+12)^{a}} \\
& +2(n-1) x^{(11+16)^{a}}+(6 m n+6 m t-22 m-6 n-12 t+36) x^{(12+13)^{a}} \\
& +(4 n t-10 n-4 t+10) x^{(12+16)^{a}}+(4 m n t-5 m t-6 n t-9 m n+ \\
& 11 m+14 n+7 t-16) x^{(16+16)^{a}}+(2 m n+2 m t-6 m-4 n-2 t \\
& +8) x^{(13+16)^{a}}
\end{aligned}
$$

Simplification leads us to the following result:

$$
\begin{aligned}
& M_{1}^{a} G_{5}(G, x)=(2 m n+2 m t-6 m-4 n-2 t+8) x^{29^{a}}+(4 m n t \\
& -5 m t-6 n t-9 m n+11 m+14 n+7 t-16) x^{32^{a}}+(4 n t-10 n-4 t \\
& +10) x^{28^{a}}+(6 m n+6 m t-22 m-6 n-12 t+36) x^{25^{a}}+(2 n-2) x^{23^{a}} \\
& +(4 m-6) x^{22^{a}}(4 m+6 t-20) x^{23^{a}}+(4 m-6) x^{20^{a}}+(4 n-8) x^{19^{a}} \\
& +(4 n t-8 n-8 t+16) x^{20^{a}}+(4 n-8) x^{14^{a}}+(2 t-4) x^{15^{a}}+2 t x^{17^{a}} \\
& +6 x^{16^{a}}+6 x^{13^{a}}+2 x^{11^{a}}+2 x^{10^{a}}+2 x^{22^{a}}+2 x^{19^{a}}+4 x^{18^{a}}+4 x^{14^{a}} .
\end{aligned}
$$

Corollary 2.5. Consider the graph of carbon graphite $C G(m, n)$ with $m ; n>2$ andt $\geq 3$, then we obtain the fifth $\mathrm{M}_{1}$-Zagreb polynomial by Theorem 2.4.

$$
\begin{aligned}
& M_{1} G_{5}(G, x)=11 x^{10}\left\{\frac{2}{11}+\left(m+\frac{14 n}{11}+\frac{7 t}{11}-\frac{9 m n}{11}\right) x^{10}\right. \\
& +\left(-\frac{5 m t}{11}-\frac{6 n t}{11}+\frac{m n t}{11}-\frac{16}{11}\right) x^{22}+\left(-\frac{6 m}{11}-\frac{4 n}{11}-\frac{2 t}{11}+\frac{2}{11} m n\right. \\
& \left.+\frac{2}{11} m t+\frac{8}{11}\right) x^{19}+\left(-\frac{10 n}{11}-\frac{4}{11} t+\frac{4}{11} n t+\frac{10}{11}\right) x^{18}+\left(\frac{2}{11} n\right. \\
& \left.-\frac{2}{11}\right) x^{17}+\left(-2 m-\frac{6 n}{11}-\frac{12 t}{11}+\frac{6 m n}{11}+\frac{36}{11}\right) x^{15}+\left(\frac{4}{11} m-\frac{4}{11}\right) x^{12} \\
& +\left(\frac{2}{11} n-\frac{2}{11}\right) x^{11}+\left(-\frac{8 n}{11}-\frac{8 t}{11}+\frac{4}{11} n t+\frac{4}{11} m+\frac{10}{11}\right) x^{10}+\left(\frac{4}{11} n\right. \\
& \left.+\frac{4}{11} t-\frac{14}{11}\right) x^{9}+\left(\frac{4}{11} m+\frac{4}{11} t-\frac{12}{11}\right) x^{8}+\frac{2}{11} t x^{7}+\frac{6}{11} x^{6}+\left(\frac{2}{11} t\right. \\
& \left.\left.-\frac{4}{11}\right) x^{5}+\left(\frac{4}{11} n-\frac{4}{11}\right) x^{4}+\frac{6}{11} x^{3}+\frac{2}{11} x\right\}
\end{aligned}
$$

Corollary 2.6. Let $\mathrm{G}$ be the graph of carbon graphite $C G(m, n)$ with $m ; n>2$ andt $\geq 3$, then 
we obtain the fifth hyper $\mathrm{M}_{1}$-Zagreb polynomial by Theorem 2.4 .

$$
\begin{aligned}
& H_{1} G_{5}(G, x)=11 x^{10}\left\{\frac{2}{11}+\left(m+\frac{14 n}{11}+\frac{7 t}{11}-\frac{9 m n}{11}\right) x^{924}\right. \\
& +\left(-\frac{6 m}{11}-\frac{4 n}{11}-\frac{2 t}{11}+\frac{2}{11} m n+\frac{2}{11} m t+\frac{8}{11}\right) x^{741} \\
& +\left(-\frac{10 n}{11}-\frac{4}{11} t+\frac{4}{11} n t+\frac{10}{11}\right) x^{684}+\left(\frac{2}{11} n-\frac{2}{11}\right) x^{629}+(-2 m \\
& \left.-\frac{6 n}{11}-\frac{12 t}{11}+\frac{6 m n}{11}+\frac{36}{11}\right) x^{525}+\left(\frac{4 m}{11}+\frac{2 n}{11}+\frac{6 t}{11}-2\right) x^{429} \\
& +\left(\frac{4}{11} m-\frac{4}{11}\right) x^{384}+\left(\frac{2}{11} n-\frac{2}{11}\right) x^{341}+\left(-\frac{8 n}{11}-\frac{8 t}{11}+\frac{4}{11} n t\right. \\
& \left.+\frac{4}{11} m+\frac{10}{11}\right) x^{300}+\left(\frac{4}{11} n+\frac{4}{11} t-\frac{14}{11}\right) x^{261}+\left(\frac{4}{11} m+\frac{4}{11} t\right. \\
& \left.-\frac{12}{11}\right) x^{224}+\frac{2}{11} t x^{189}+\frac{6}{11} x^{156}+\left(\frac{2}{11} t-\frac{4}{11}\right) x^{125}+\left(\frac{4}{11} n\right. \\
& \left.\left.-\frac{4}{11}\right) x^{96}+\frac{6}{11} x^{69}+\frac{2}{11} x^{21}\right\}
\end{aligned}
$$

Theorem 2.5. Consider the graph of carbon graphite $C G(m, n)$ with $m ; n>2$ and $t \geq 3$, then general fifth $\mathrm{M}_{2}$-Zagreb polynomial is equal to:

$$
\begin{aligned}
& M_{2}^{a} G_{5}(G, x)=(4 m+6 t-16) x^{80^{a}}+(2 n-2) x^{104^{a}}+(2 n-2) x^{132^{a}} \\
& +(2 n-2) x^{176^{a}}+(2 t-4) x^{56^{a}}+(4 n-8) x^{48^{a}}+(2 t-8) x^{84^{a}} \\
& +(4 n t-8 n-8 t+16) x^{96^{a}}+(4 n-8) x^{88^{a}}+2 t x^{70^{a}}+2 x^{117^{a}} \\
& +2 x^{90^{a}}+2 x^{64^{a}}+4 x^{77^{a}}+2 x^{63^{a}}+4 x^{49^{a}}+2 x^{60^{a}}+6 x^{40^{a}} \\
& +2 x^{30^{a}}+2 x^{25^{a}}+(2 m n+2 m t-6 m-4 n-2 t+8) x^{208^{a}}+(4 m n t \\
& -5 m t-6 n t-9 m n+11 m+14 n+7 t-16) x^{256^{a}}+(4 n t-10 n-4 t \\
& +10) x^{192^{a}}+(6 m n+6 m t-22 m-6 n-12 t+36) x^{156^{a}} \\
& +(4 m-6) x^{100^{a}}+(4 m-6) x^{120^{a}}+(4 m+6 t-20) x^{130^{a}}
\end{aligned}
$$

Proof. Let $\mathrm{G} \approx \mathrm{CG}(\mathrm{m}, \mathrm{n})$ be a graph of carbon graphite. The above result can be proved using Table 1 and Equation 5 in the following computation. The general fifth Zagreb polynomial is computed as below:

$$
\begin{aligned}
& M_{2}^{a} G_{5}(G, x)=\sum_{u v \in E(G)} x^{\left(S_{G}(u) \times S_{G}(v)\right)^{a}}=(2) x^{(5 \times 5)^{a}} \\
& +(2) x^{(5 \times 6)^{a}}+(6) x^{(5 \times 8)^{a}}+4(n-2) x^{(6 \times 8)^{a}}+(2) \\
& x^{(6 \times 10)^{a}}+(4) x^{(7 \times 7)^{a}}+2(t-2) x^{(7 \times 8)^{a}}+(2) x^{(7 \times 9)^{a}} \\
& +(2 t) x^{(7 \times 10)^{a}}+(4) x^{(7 \times 11)^{a}}+4(t-2) x^{(7 \times 12)^{a}}+(2) \\
& x^{(8 \times 8)^{a}}+4(m+t-4) x^{(8 \times 10)^{a}}+4(n-2) x^{(8 \times 11)^{a}} \\
& +4(n t-2 n-2 t+4) x^{(8 \times 12)^{a}}+2(n-1) x^{(8 \times 13)^{a}}+(2) \\
& x^{(9 \times 10)^{a}}+(2) x^{(9 \times 13)^{a}}+(4 m-6) x^{(10 \times 10)^{a}}+(4 m-6) \\
& x^{(10 \times 12)^{a}}+(4 m+6 t-20) x^{(10 \times 13)^{a}}+2(n-1) x^{(11 \times 12)^{a}} \\
& +2(n-1) x^{(11 \times 16)^{a}}+(6 m n+6 m t-22 m-6 n-12 t+36) x^{(12 \times 13)^{a}} \\
& +(4 n t-10 n-4 t+10) x^{(12 \times 16)^{a}}+(4 m n t-5 m t-6 n t-9 m n+ \\
& 11 m+14 n+7 t-16) x^{(16 \times 16)^{a}}+(2 m n+2 m t-6 m-4 n-2 t \\
& +8) x^{(13 \times 16)^{a}}
\end{aligned}
$$

After an easy calculation, we got:

$$
\begin{aligned}
& M_{2}^{a} G_{5}(G, x)=(4 m+6 t-16) x^{80^{a}}+(2 n-2) x^{104^{a}}+(2 n-2) x^{132^{a}} \\
& +(2 n-2) x^{176^{a}}+(2 t-4) x^{56^{a}}+(4 n-8) x^{48^{a}}+(2 t-8) x^{84^{a}} \\
& +(4 n t-8 n-8 t+16) x^{96^{a}}+(4 n-8) x^{88^{a}}+2 t x^{70^{a}}+2 x^{117^{a}} \\
& +2 x^{90^{a}}+2 x^{64^{a}}+4 x^{77^{a}}+2 x^{63^{a}}+4 x^{49^{a}}+2 x^{60^{a}}+6 x^{4^{a}} \\
& +2 x^{30^{a}}+2 x^{25^{a}}+(2 m n+2 m t-6 m-4 n-2 t+8) x^{208^{a}}+(4 m n t \\
& -5 m t-6 n t-9 m n+11 m+14 n+7 t-16) x^{256^{a}}+(4 n t-10 n-4 t \\
& +10) x^{192^{a}}+(6 m n+6 m t-22 m-6 n-12 t+36) x^{156^{a}} \\
& +(4 m-6) x^{100^{a}}+(4 m-6) x^{120^{a}}+(4 m+6 t-20) x^{130^{a}}
\end{aligned}
$$

Corollary 2.7. Consider the graph of carbon graphite $C G(m, n)$ with $m ; n>2$ andt $\geq 3$, then we obtain the fifth $\mathrm{M}_{2}$-Zagreb polynomial by Theorem 2.5.

$$
\begin{aligned}
& M_{2} G_{5}(G, x)=x^{25}(2+(4 m n t-5 m t-6 n t-9 m n+11 m \\
& +14 n+7 t-16) x^{231}+(4 n-8) x^{63}+(4 t-8) x^{59}+(4 m+4 t-16) x^{55} \\
& +(2 t-4) x^{31}+(4 n-8) x^{23}+(2 m n+2 m t-6 m-4 n-2 t+8) x^{183} \\
& +(4 n t-10 n-4 t+10) x^{167}+(2 n-2) x^{151}+(-6 n-12 t+6 m n+6 m t \\
& -2 m+36) x^{131}+(2 n-2) x^{107}+(4 / 11 m+6 t-20) x^{105}+(4 m-6) x^{195} \\
& +(2 n-2) x^{79}+(4 m-6) x^{75}+(4 / 11 n t-8 n-8 t+16) x^{71}+2 x^{92} \\
& +2 x^{65}+2 x^{39}+4 x^{52}+2 x^{38}+4 x^{24}+2 x^{35}+6 x^{15}+2 x^{5}+2 t x^{45}
\end{aligned}
$$

Corollary 2.8. Let $\mathrm{G}$ be the graph of carbon graphite $C G(m, n)$ with $m ; n>2$ andt $\geq 3$, then 
we obtain the fifth hyper $\mathrm{M}_{2}$-Zagreb polynomial by Theorem2.5.

$$
\begin{aligned}
& H_{2} G_{5}(G, x)=x^{625}(2+(4 m n t-5 m t-6 n t-9 m n+11 m+14 n \\
& +7 t-16) x^{64911}+(4 n-8) x^{1679}+(4 t-8) x^{6431}+(4 m+4 t-16) x^{5775} \\
& +(2 t-4) x^{2511}+(4 n-8) x^{7119}+(2 m n+2 m t-6 m-4 n-2 t+8) x^{42639} \\
& +(4 n t-10 n-4 t+10) x^{36239}+(2 n-2) x^{30351}+(-6 n-12 t+6 m n+6 m t \\
& -2 m+36) x^{23711}+(2 n-2) x^{16799}+(4 m+6 t-20) x^{16275}+(4 m-6) x^{13775} \\
& +(2 n-2) x^{10191}+(4 m-6) x^{9375}+(4 n t-8 n-8 t+16) x^{8591}+2 x^{13064} \\
& +2 x^{7475}+2 x^{3471}+4 x^{5304}+2 x^{3344}+4 x^{1776}+2 x^{2975}+6 x^{975}+2 x^{275} \\
& +2 t x^{4275}
\end{aligned}
$$

\section{Conclusion}

In this paper, we have computed the general fifth M-Zagreb indices $M_{1}{ }^{a} G_{5}(G), M_{2}{ }^{a} G_{5}(G)$ and fifth M-Zagreb indices $M_{1} G_{5}(G), M_{2} G_{5}(G)$, $\mathrm{HM}_{1} \mathrm{G}_{5}(\mathrm{G})$ and $\mathrm{HM}_{2} \mathrm{G}_{5}(\mathrm{G})$. Also, we have computed fifth $\mathrm{M}_{3}$-Zagreb index $\mathrm{M}_{3} \mathrm{G}_{5}(\mathrm{G})$. In the end, we have given the exact general fifth M-Zagreb $\quad \mathrm{M}_{1}{ }^{\mathrm{a}} \mathrm{G}_{5}(\mathrm{G} ; \mathrm{x}), \quad \mathrm{M}_{2}{ }^{\mathrm{a}} \mathrm{G}_{5}(\mathrm{G} ; \mathrm{x}) \quad$ and particularly fifth M-Zagreb $\mathrm{M}_{1} \mathrm{G}_{5}(\mathrm{G} ; \mathrm{x})$, $\mathrm{M}_{2} \mathrm{G}_{5}(\mathrm{G} ; \mathrm{x}), \mathrm{HM}_{1} \mathrm{G}_{5}(\mathrm{G} ; \mathrm{x})$ and $\mathrm{HM}_{2} \mathrm{G}_{5}(\mathrm{G} ; \mathrm{x})$ of carbon graphite for the first time.

\section{Acknowledgements}

We send our gratitude to the anonymous referees and editors for their valuable suggestions and comments to improve this paper.

\section{Orcid:}

Muhammad Naeem: https://orcid.org/00000002-8132-1580

\section{References}

[1] A.Q. Baig, M. Imran, W. Khalid, M. Naeem, Can. J. Chem, 2017, 95, 674-686.

[2] M.R. Farahani, J. Math. Nanosci., 2012, 2, 15-20.

[3] W. Gao, W.F. Wang, J. of Diff. Equ. \& App., 23, 2016, 1-10.
[4] A. Graovac, M. Ghorbani, M.A. Hosseinzadeh, J. of Math. Nanosci., 2011, 1, 33-42.

[5] M.N. Husin, R. Hasni, N.E. Arif, M. Imran, Molecules, 2016, 21, 821.

[6] M. Imran, M. Naeem, A. Qadair Baig, M. K. Siddiqui, M.A. Zahid, W. Gao, J. of Dis. Math. Sci. and Cry., 2019, 22, 1215-1228.

[7] M. Imran, A.A.E. Abunamous, D. Adi, S.H. Rafique, A.Q. Baig, M.R. Farahani, J. of Dis. Math. Sci. and Cry., 2019, 22, 1199-1213.

[8] V.R. Kulli, Intern. J. Fuzzy Math. Arch. 2017, 13, 99-103.

[9] V.R. Kulli, College Graph Theory, Vishwa International Publications, Gulbarga, India, 2012.

[10] V.R. Kulli, Int. J. Math. Arch., 2017, 8, 103109.

[11] J.B. Liu, A.Q. Baig, W. Khalid, M.R. Farahani, Com. Ren. de l'Acad. bulg. des Sci., Tom., 2018, 71, 10-21.

[12] H. Yang, M. Naeem, A.Q. Baig, H. Shaker, M.K. Siddiqui. J. Dis. Math. Sci. and Cryp., 2019, 22, 1177-1187.

[13] M.A. Zahid, M. Naeem, A.Q. Baig, Wei Gao, Utilit. Math., 2018, 109, 263-270.

How to cite this article: Wei Gao, Muhammad Naeem*, Abdul Qudair Baig, JiaBao Liu. General fifth M-Zagreb indices and fifth M-Zagreb polynomials of carbon graphite. Eurasian Chemical Communications, 2020, 2(5), 634-640. Link: http://www.echemcom.com/article_104189 .html 